\title{
W7-X Neutral-Beam-Injection: Selection of the NBI source positions for experiment start-up
}

\author{
Norbert Rust, Bernd Heinemann, Boris Mendelevitch, Alan Peacock, Michael Smirnow \\ Max-Planck-Institut für Plasmaphysik, Association EURATOM-IPP, D-85748 Garching
}

\begin{abstract}
The stellarator W7-X will be equipped with two Neutral-Beam-Injector (NBI) boxes for balanced injection. Each NBI box has 2 tangential and 2 radial source positions. For the experimental start-up phase each NBI box will be only equipped with 2 ion sources. For the selection of the initial 2 NBI source positions per box three physical aspects were examined (transmission and duct power deposition, shine through and heating efficiency)

Using hydrogen injection the heating power to the plasma under typically planned conditions should be 1.3MW for the tangential sources and 1.1MW for the radial sources (Deuterium: $2 \mathrm{MW}$ for the tangential sources, $1.8 \mathrm{MW}$ for the radial sources). The tangential source positions all have similar heating efficiencies. One of them suffers from the lowest duct transmission (highest power-load to the duct). The same source hits a component with a low power-load capability. The W7-X inner wall design will be modified in order to enhance the maximum power-load capability of that component. For the radial source positions there is no clear physics advantage of one position over the other. Taking all aspects into consideration the decision was made to use one tangential source and one radial source per box during the experimental start-up phase.
\end{abstract}

Keywords: W7-X, Neutral-Beam-Injection, transmission, shine-through, heat efficiency

\section{Introduction}

W7-X will be equipped with two Neutral-Beam Injector-Boxes (NI20 and NI21) for balanced injection. They are replicas of the ASDEX-Upgrade NBI-system [1] and each of them has the capacity to be equipped with 4 PINI-sized [2] RF ion sources [3] of 2.5MW (D ${ }^{0}$, $60 \mathrm{kV})$ or $1.7 \mathrm{MW}\left(\mathrm{H}^{0}, 55 \mathrm{kV}\right)$ neutral power per source and a maximum pulse-length of 10s. For the experimental start-up phase each NBI box will be only equipped with 2 ion sources. Both NBI boxes connect to torus module 2. Because of the good confinement for fast ions in $\mathrm{W} 7-\mathrm{X}$ it is possible to use nearly radial injection geometry with an angle of $7.5^{\circ}$ with respect to the duct

Fig. 1 shows the positions of the NBI sources and a sketch of the beam axes for the two W7-X NBI boxes arranged $\pm 4.1^{\circ}$ to the box axes, so there are tangential and radial neutral beam sources with different physical characteristics. Because of the symmetry of the W7-X magnetic field, the balanced injection has a quasi symmetry. Source 1 of NI20 has similar physical characteristics to source 5 of NI21, etc. In addition Fig. 1 shows in red a sketch of the shape of the W7-X NBI duct at its narrowest position.

\section{Transmission through the duct}

The superconducting coils of the W7-X stellarator limit the size and shape of the duct. There are two constrictions caused by the planar and nonplanar W7-X coils near the duct. Fig 2 illustrates the W7-X NBI duct.

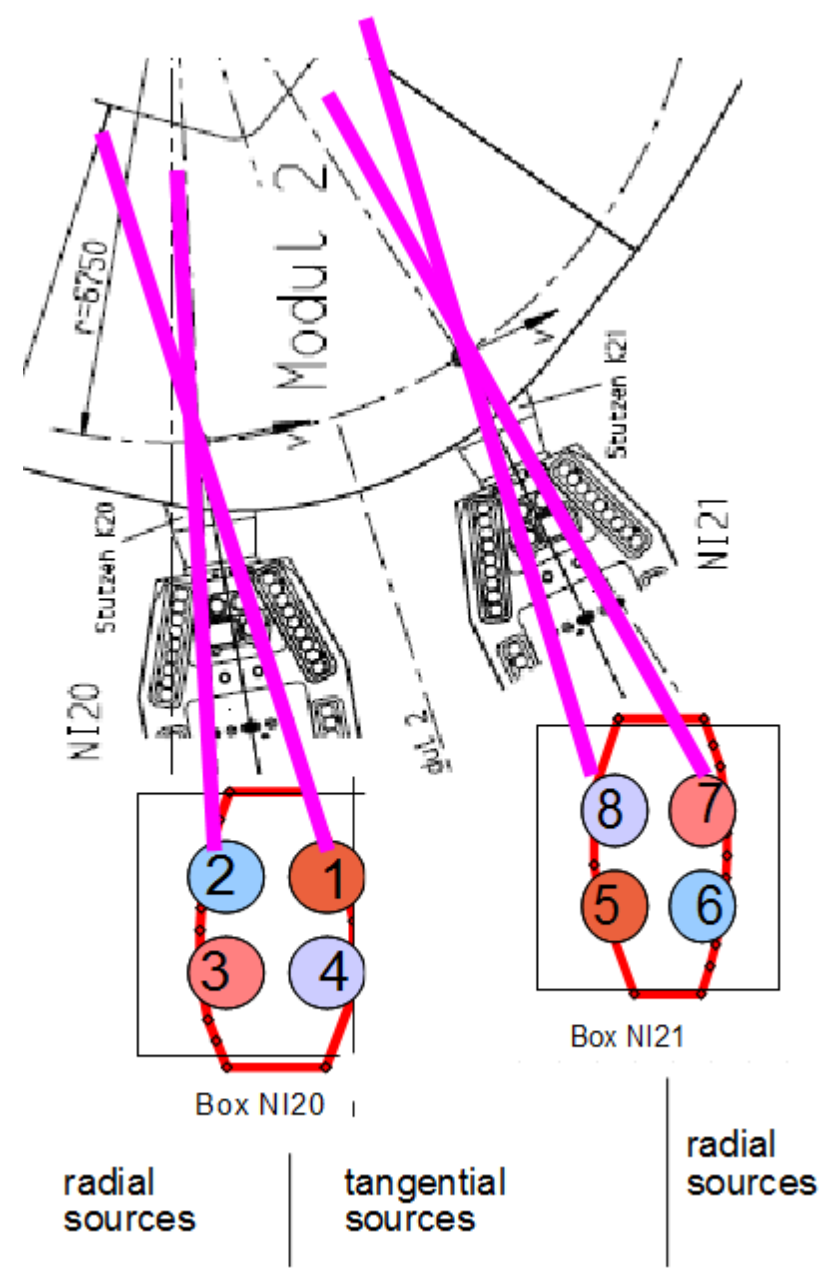

Fig.1 W7-X NBI beam geometry and source positions (back view towards the torus) 


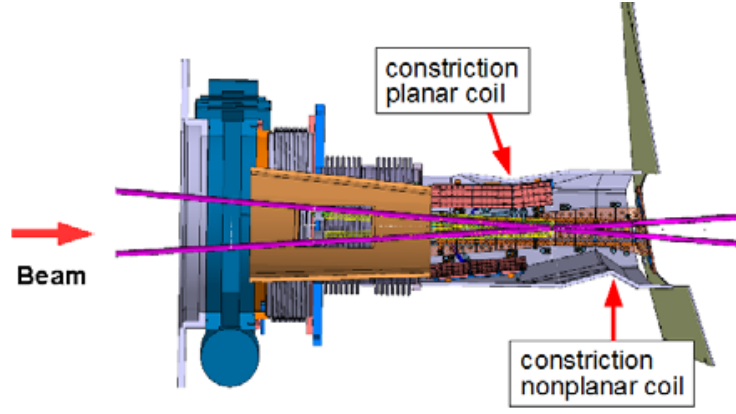

Fig.2 W7-X NI21 NBI duct with two constrictions due to constrictions with W7-X superconducting magnetic field coils

The W7-X NBI duct is covered with cooled copper plates on the left part of Fig 2 and with graphite or CFC elements mounted on a cooled CuCrZr construction in the area of the constrictions.

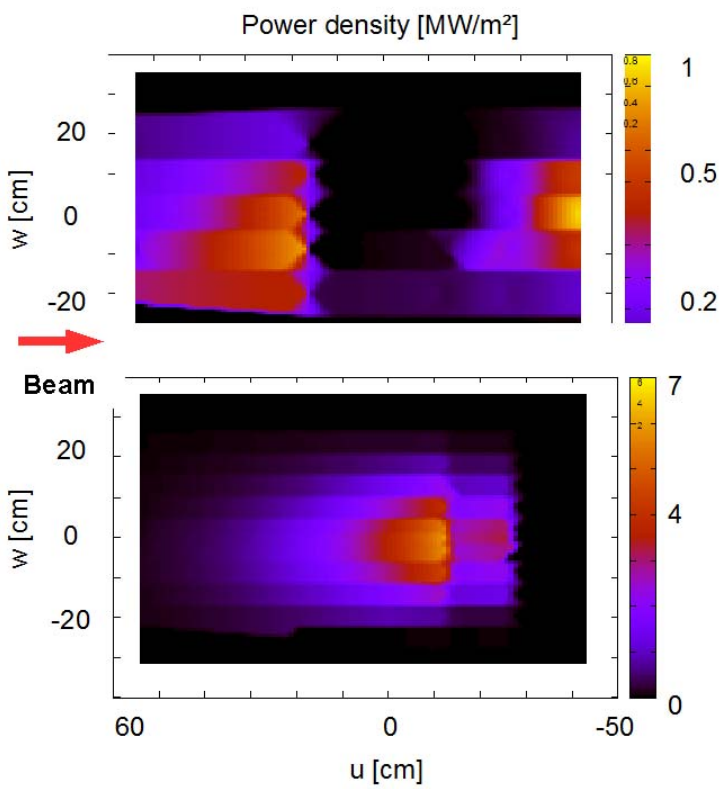

Fig.3 W7-X NBI power-load to the duct protection top: NI21 left side of the duct, color scale $1 \mathrm{MW} / \mathrm{m}^{2}$ bottom: NI21 right side of the duct, color scale $7 \mathrm{MW} / \mathrm{m}^{2}$ (deuterium $60 \mathrm{kV}, 1^{\circ}$ divergence)

The transmission and the power-load to the duct were calculated using the in-house code DensB [4]. The calculated transmission is given in Table 1 . The source positions 1 and 5 suffer most from both constrictions. This is related to the highest power-load to the duct presented in Fig. 3 for deuterium injection. Note the difference in the color scale between the top and the bottom. The highest power-load to the duct $\left(6.3 \mathrm{MW} / \mathrm{m}^{2}\right)$ is near the constriction with the nonplanar coils and has a gradient of the beam power of $1.3 \mathrm{MW} / \mathrm{m}^{2} / \mathrm{cm}$. This does not limit a 10s NBI pulse, but no further contraction of the duct geometry is possible without limiting the NBI pulse seriously. This high power-load is caused by lower duct transmission of the tangential sources.
Table 1: transmission through the duct for different sources as calculated by the program DensB

\begin{tabular}{l|cccc} 
NI20 & Source 1 & Source 2 & Source 3 & Source 4 \\
NI21 & Source 5 & Source 6 & Source 7 & Source 8 \\
\hline geometry & tangential & radial & radial & tangential \\
transmission & $79 \%$ & $89 \%$ & $91 \%$ & $84 \%$
\end{tabular}

\section{Power-load to the inner wall}

NBI shine-through is beam that strikes the inner wall of the W7-X plasma vessel even with a plasma present. The geometry of the inner vessel in the region of the NBI beam footprint is quite complex. The W7-X heatshield, divertor, baffle, and standard wall panel elements are affected. Fig. 4 shows the contour lines of the power-load and the position of the maximum powerdensity for 2 sources per box and for 4 sources per box. As originally designed the low maximal power-load capabilities $\left(100 \mathrm{~kW} / \mathrm{m}^{2}\right)$ of the standard wall panels hit by beams from source 1 and 5 would forbid the use of this source. In the initial W7-X operational phase many parts of the inner wall will have no water cooling. This would have restricted the NBI pulse length to $1.7 \mathrm{~s}$ as compared to 4.1s with water cooling (hydrogen, density $4 \mathrm{E} 19 / \mathrm{m}^{3}$ ). As a result of both the presented calculations and other thermal calculations the W7-X inner wall hit by the NBI shine-through will now be supplied with water cooling and the wall panel will be replaced by a new construction (CFC) with a significantly higher power-load capability.
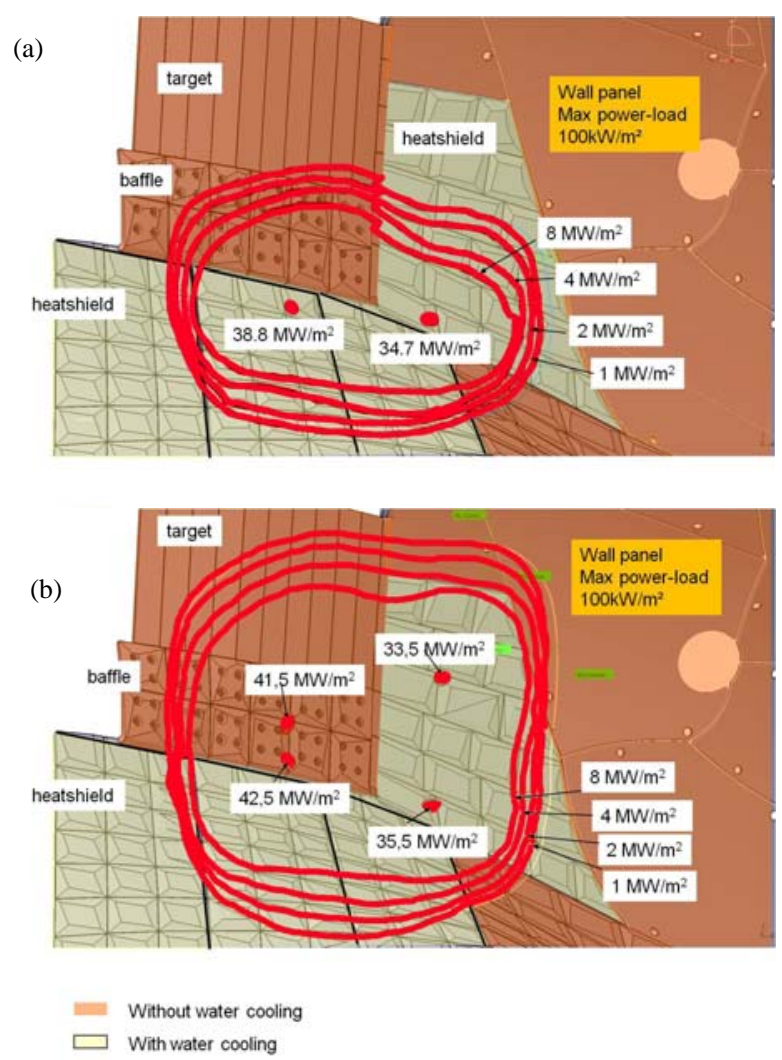

Fig.4 NI21 Power-load to the inner wall (a) source 7 and source 8 (b) 4 sources per box (deuterium $60 \mathrm{kV}$ divergence $=1^{\circ}$, no plasma) 
For beam injection without plasma the maximal power density deposited on the inner wall is between $39 \mathrm{MW} / \mathrm{m}^{2}$ (2 sources per box) and $43 \mathrm{MW} / \mathrm{m}^{2}$ (4 sources per box). This requires a diagnostic for observation of the surface temperature of the inner wall coupled with a quick termination of the NBI in the case of the surface temperature exceeding the maximum allowed value of $1800^{\circ} \mathrm{C}$ (maximum pulselengh without plasma: 500ms).

\section{Shine-through density dependence - heating efficiency}

Without plasma present the thermal power density to the inner wall is very high (up to $43 \mathrm{MW} / \mathrm{m}^{2}$ ). It decreases significantly with rising plasma density. Finally the heating efficiency is an essential criterion for the choice of the initial sources. The density dependence and the heating efficiency were calculated using the W7 code [5].

As already mentioned there are tangential and radial source positions. There is a top- bottom asymmetry of the different sources, because of the shape of the magnetic field penetrated by the neutral-beams. Fig 5 shows the side view of the beam axes of all sources.

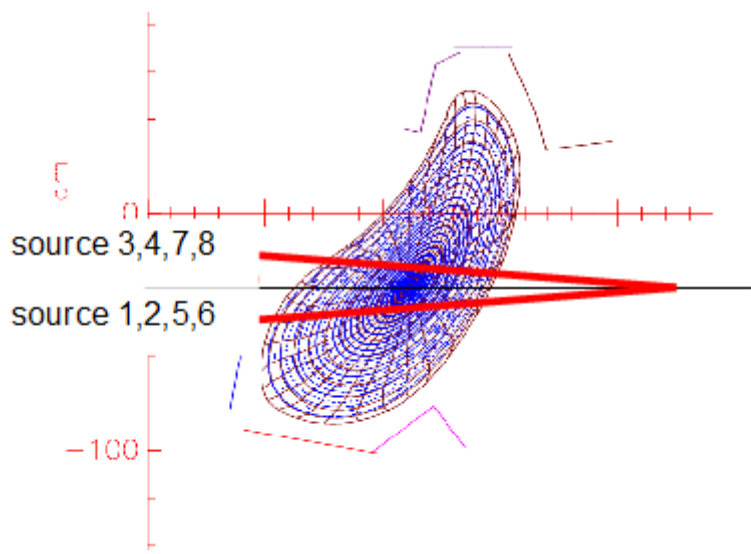

Fig. 5 side view of the different beams in the W7-X magnetic field

Fig. 6 shows the density dependency of the shinethrough for hydrogen and deuterium. The shine-through is independent of co- or counter-injection making Fig. 6 valid for both NI20 and NI21. Without plasma the power load for deuterium is higher because of the higher neutral-beam power during deuterium operation. With rising density the decline in the case of deuterium is sharper than in the case of hydrogen. For a central density of $1 \mathrm{E} 20 / \mathrm{m}^{3}$ the maximal power-load to the wall is $3.5 \mathrm{MW} / \mathrm{m}^{2}$ for hydrogen and $1.7 \mathrm{MW} / \mathrm{m}^{2}$ for deuterium and the shine-through is about $10 \%$ in the case of hydrogen compared to less than $4 \%$ in the case of deuterium (4 sources per box). As for the high powerload to the W7-X inner wall, there is no NBI pulse length limitation for densities larger than $1 \mathrm{E} 20 / \mathrm{m}^{3}$ (hydrogen) and 8E19/m³ (deuterium).

Source 1 has the steepest decline of the shine-through with density. Source 1 is a tangential source and its beam has a longer distance in the plasma compared with the second tangential source (4) because of the top- bottom asymmetry. The radial sources 2 and 3 show a similar difference due to the top- bottom asymmetry. While the shine-through of source 3 is the largest of all 4 sources, the shine-through of source 2 is close to the tangential source 4.
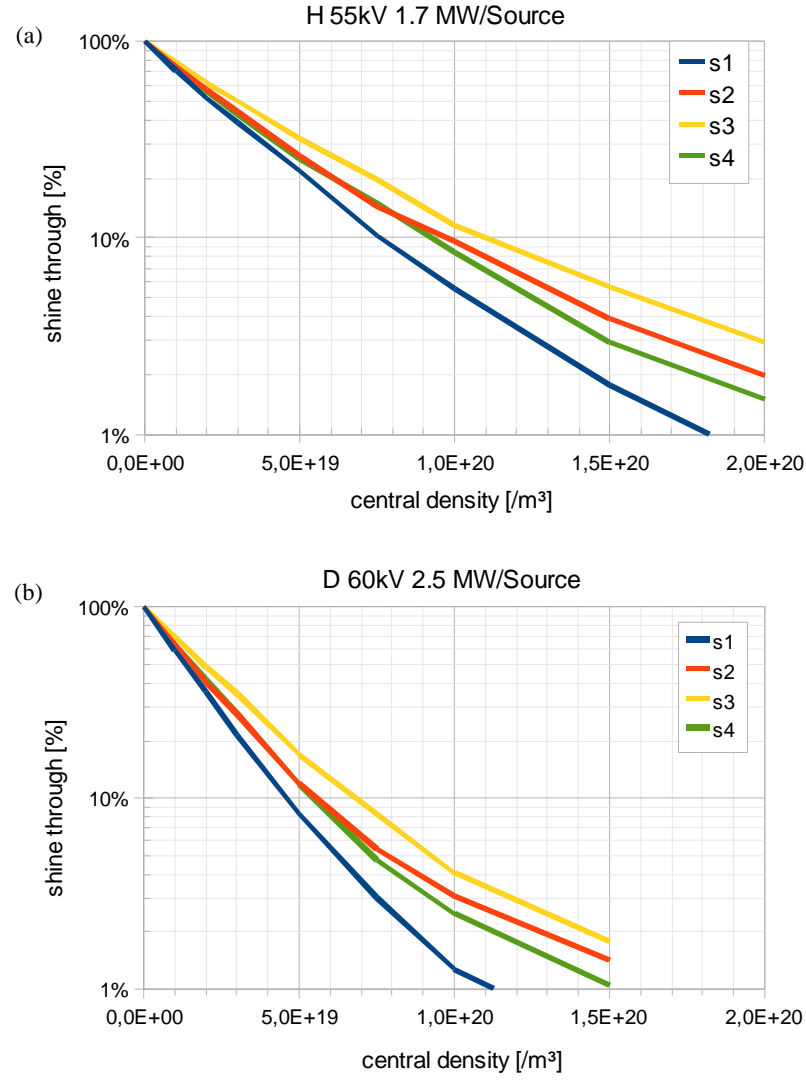

Fig.6 NBI shine-through vs. plasma density for different sources a) hydrogen $55 \mathrm{kV}$ b) deuterium $60 \mathrm{kV}$

Lastly the most interesting topic for a NBI system is the heating power. The heating power depends on the transmission through the duct, the power deposition to the plasma and the slowing-down of the fast ions. In addition there is density dependence in both the power deposition profile and the heating profile of the fast ions generated by the NBI. For example, the power deposition profile is centrally peaked for a central density of $1 \mathrm{E} 20 / \mathrm{m}^{3}$ in the case of hydrogen injection and for central density of $7.5 \mathrm{E} 19 / \mathrm{m}^{3}$ in the case of deuterium injection. For density of $1.5 \mathrm{E} 20 / \mathrm{m}^{3}$ the power deposition profile of deuterium injection is flat and with density decreasing further it becomes off-axes peaked.

Fig 7 shows a comparison of the different source positions for a central density of $1 \mathrm{E} 20 / \mathrm{m}^{3}$ and hydrogen $(55 \mathrm{kV})$. The power deposition is the injected power minus the shine-through. The heating power is the power deposition minus orbit loss. Sources 1 and 5 have the lowest transmission through the duct. The radial source positions show the best transmission. For the power deposition there should be an advantage for the tangential sources, but lower transmission negates this. The shine-through and the power load to the inner wall are significantly higher for the radial sources. The orbit loss of the fast ions generated by radial sources is higher 


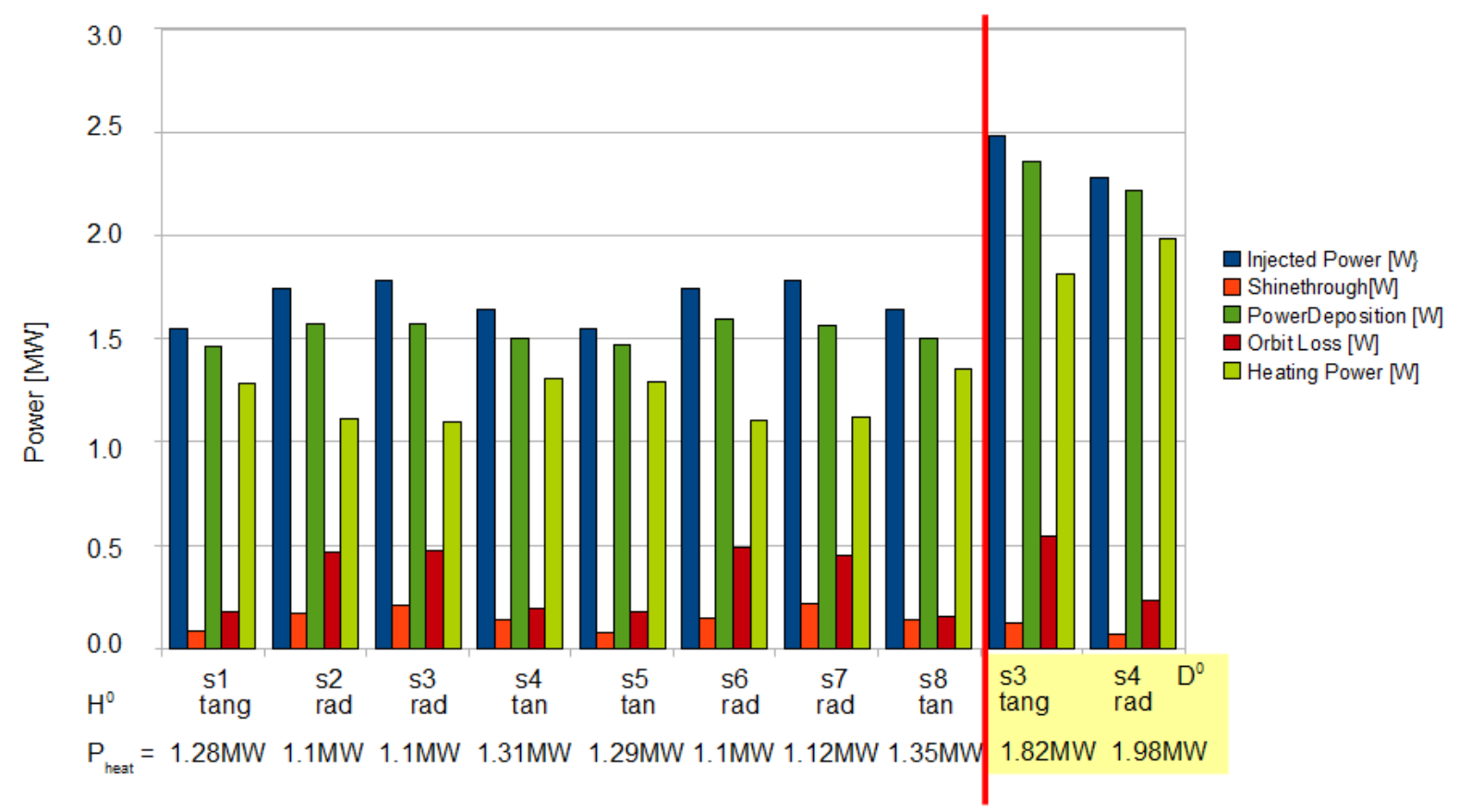

Fig.7 comparison of the different source positions for a central density of $1 \mathrm{E} 20 / \mathrm{m}^{3}$ : Sources 1-8 hydrogen $55 \mathrm{kV}$, source 3 and 4 deuterium $60 \mathrm{kV}$

too. So the heating power is higher for the tangential sources. There is little difference between co-injection (sources 1-4) and counter-injection (sources 5-8). For comparison, the right side of Fig. 7 shows the results for deuterium injection $(60 \mathrm{kV})$ for sources 3 and 4 . The heating power using deuterium is significantly higher but both shine-through and the orbit loss are on the same level as with hydrogen injection.

\section{Final selection of the NBI source positions for experiment start-up}

Table 2 shows a summary of all the analyzed physical aspects.

All the tangential source positions (source 1,4,5 and 8 ) have about the same heating power. But source 1 and 5 show the worst transmission through the duct. This leads to the highest power-load to the duct protection. In addition the same sources hit the wall panel on the W7$\mathrm{X}$ inner wall that will be replaced. So the tangential source positions 4 and 8 are used for experiment startup.

Table 2: summary and comparison of the analyzed physical aspects for the different source positions of the source specific plasma heating characteristics

\begin{tabular}{l|cccc} 
NI20 & Source 1 & Source 2 & Source 3 & Source 4 \\
NI21 & Source 5 & Source 6 & Source 7 & Source 8 \\
\hline geometry & tangential & radial & radial & tangential \\
transmission & $79 \%$ & $89 \%$ & $91 \%$ & $84 \%$ \\
powerload duct & high & low & low & high \\
powerload W7-X inner wall & hits & OK & OK & OK \\
& wall pannel & & & \\
\hline heating power $(\mathrm{H} 255 \mathrm{kV})$ & $1.3 \mathrm{MW}$ & $1.1 \mathrm{MW}$ & $1.1 \mathrm{MW}$ & $1.3 \mathrm{MW}$
\end{tabular}

For the radial source positions it is not possible to get a clear decision from the analyzed physical aspects. The heating power is about the same and there is no advantage or disadvantage for either of the two positions. The decision was made to use source 3 and 7 for experiment start-up due to the ease of mechanical assembly.

\section{Conclusion}

Based on the analysis of different physical properties of different NBI source positions (transmission and duct power deposition, shine through and heating efficiency) the decision was made to use one tangential and radial source for initial W7-X operation. Additionally the W7$X$ inner wall hit by the NBI shine-through will be improved (water cooling, elimination of low power-load elements), so later an upgrade of the NBI system to 4 sources per pox will be possible and the additional severe pulse length limitation due to the absence of water cooling will be avoided.

[1] A. Stäbler, J.-H. Feist, E. Speth, et al., Fusion Technology (1988) 620

[2] G. Duesing, H. Altmann, H. Falter, et al., Fusion Technology 11 (1987) 163

[3] E. Speth, M. Ciric, J.H. Feist, et al. Fusion Engineering and Design 46 (1999) 383

[4] N. Rust, H. Greuner, B. Heinemann, M. Kick, R. Riedl, E. Speth, Soft 2004

[5] M. Schmidt, Y. Turkin, A. Werner, 31st EPS Conference on Plasma Phys. 28G (2004) P-1.120 